\title{
A Note on the Characterization of Zero-Inflated Poisson Model
}

\author{
G. Nanjundan, Sadiq Pasha \\ Department of Statistics, Bangalore University, Bangalore, India \\ Email: nanzundan@gmail.com
}

Received 14 March 2015; accepted 16 April 2015; published 20 April 2015

Copyright (C) 2015 by authors and Scientific Research Publishing Inc.

This work is licensed under the Creative Commons Attribution International License (CC BY).

http://creativecommons.org/licenses/by/4.0/

(c) (i) Open Access

\begin{abstract}
Zero-Inflated Poisson model has found a wide variety of applications in recent years in statistical analyses of count data, especially in count regression models. Zero-Inflated Poisson model is characterized in this paper through a linear differential equation satisfied by its probability generating function [1] [2].
\end{abstract}

\section{Keywords}

Zero-Inflated Poisson Model, Probability Generating Function, Linear Differential Equation

\section{Introduction}

A random variable $X$ is said to have a zero-inflated Poisson distribution if its probability mass function is given by

$$
\begin{aligned}
p(x ; \theta, \varphi) & = \begin{cases}\varphi+(1-\varphi) \mathrm{e}^{-\theta}, & x=0 \\
(1-\varphi) \frac{\mathrm{e}^{-\theta} \theta^{x}}{x !}, & x=1,2, \cdots\end{cases} \\
= & \varphi p_{0}(x)+(1-\varphi) p_{1}(x), \quad 0<\varphi<1
\end{aligned}
$$

where $p_{0}(x)=\left\{\begin{array}{ll}1, & x=0 \\ 0, & x \neq 0\end{array}\right.$ and $\quad p_{1}(x)=\frac{\mathrm{e}^{-\theta} \theta^{x}}{x !}, \quad x=0,1,2, \cdots, \quad \theta>0$.

Thus, the distribution of $X$ is a mixture of a distribution degenerate at zero and a Poisson distribution with mean $\theta$. 


\section{Probability Generating Function}

The probability generating function (pgf) of $X$ is given by

$$
\begin{aligned}
f(s) & =E\left(s^{X}\right) \\
& =\sum_{k=0}^{\infty} p_{k} s^{k} \\
& =\varphi+(1-\varphi) \mathrm{e}^{-\theta}+(1-\varphi) \mathrm{e}^{-\theta} \sum_{k=1}^{\infty} \frac{(\theta s)^{k}}{k !}, \quad 0<s<1 . \\
f(s) & =\varphi+(1-\varphi) \mathrm{e}^{\theta(s-1)} .
\end{aligned}
$$

\section{Characterization}

Let $X$ be a non-negative integer valued random variable with $0<P(X=0)<1$ and the pgf $f(s)$. Then, the distribution of $X$ is zero-inflated Poisson if and only if $f(s)=a+b f^{\prime}(s)$, where $0<a<1, b$ are constants and $f^{\prime}(s)$ is the first derivative of $f(s)$.

Proof:

1) Suppose that $X$ has a zero-inflated Poisson distribution specified in (1.1). Then the pgf of $X$ is given by

$$
f(s)=\varphi+(1-\varphi) \mathrm{e}^{\theta(s-1)}
$$

On differentiation, we get

$$
\begin{aligned}
f^{\prime}(s) & =(1-\varphi) \mathrm{e}^{\theta(s-1)} \theta \\
& =\theta\{f(s)-\varphi\}, \\
f(s) & =\varphi+\frac{1}{\theta} f^{\prime}(s) .
\end{aligned}
$$

Hence $f(s)$ satisfies the linear differential equation

$$
f(s)=a+b f^{\prime}(s)
$$

2) Suppose that the pgf $f(s)$ of $X$ satisfies

$$
f(s)=a+b f^{\prime}(s)
$$

If $b=0$, then $f(s)=a$ and in turn $f(0)=f(1)=a$. By the property of the pgf, $f(1)=1=a$. But $f(0)=P(X=0)=a$, which is not possible because $P(X=0)<1$.

Therefore $b \neq 0$.

3) The Linear Differential Equation

The linear differential equation $f(s)=a+b f^{\prime}(s)$ is of the form

$$
\frac{\mathrm{d} y}{\mathrm{~d} x}+P y=Q
$$

where $P$ and $Q$ are functions of $x$.

Then its solution is given by

$$
y \mathrm{e}^{\int P \mathrm{~d} x}=\int Q \mathrm{e}^{\int P \mathrm{~d} x} \mathrm{~d} x+c
$$

where $c$ is an arbitrary constant. 
Here

$$
\begin{gathered}
b f^{\prime}(s)-f(s)=-a \\
\Rightarrow f^{\prime}(s)-\frac{1}{b} f(s)=-\frac{a}{b} .
\end{gathered}
$$

Hence $P=-\frac{1}{b}, Q=-\frac{a}{b}$.

Therefore the solution of the Equation (2) is given by

$$
f(s)=a+c \mathrm{e}^{s / b} .
$$

We now extract the probabilities $P(X=k)=p_{k}, \quad k=0,1,2, \cdots \quad$ using the above solution.

Since $f(s)$ is a pgf, $p_{k}=\frac{f^{(k)}(0)}{k !}$, where $f^{(k)}(s)$ is the $k$-th derivative of $f(s)$.

We get

$$
p_{0}=a+c, p_{1}=c \frac{1}{b}, \quad p_{2}=c \frac{1}{2 !} \frac{1}{b^{2}}, \text { and so on. }
$$

Now,

$$
f(s)=\sum_{k=0}^{\infty} p_{k}=a+c \mathrm{e}^{1 / b}
$$

Since $f(1)=1$, it is easy to see that $c=(1-a) \mathrm{e}^{-1 / b}$, We have

$$
p_{k}= \begin{cases}a+(1-a) \mathrm{e}^{-1 / b}, & k=0 \\ (1-a) \frac{\mathrm{e}^{-1 / b}(1 / b)^{k}}{k !}, & k=1,2,3, \cdots\end{cases}
$$

with $\varphi=a$ and $\theta=1 / b$.

Therefore $X$ has the pgf specified in Equation (1).

\section{References}

[1] Nanjundan, G. (2011) A Characterization of the Members of a Subfamily of Power Series Distributions. Applied mathematics, 2, 750-751. http://dx.doi.org/10.4236/am.2011.26099

[2] Nanjundan, G. and Ravindra Naika, T. (2012) An Asymptotic Comparison of the Maximum Likelihood and the Moment Estimators in a Zero-Inflated Poisson Model. Applied mathematics, 3, 610-617.

http://dx.doi.org/10.4236/am.2012.36095 